\title{
Complex viscosity behavior and cluster formation in attractive colloidal systems
}

\author{
F. Mallamace ${ }^{1,2}$, S. H. Chen ${ }^{1}$, A.Coniglio ${ }^{3,6}$, L. de Arcangelis ${ }^{4,6}$, E. Del Gado ${ }^{5,6}$ and A. Fierro ${ }^{3,6}$ \\ ${ }^{1}$ Department of Nuclear Engineering, Massachusetts Institute of Technology, Cambridge MA 02139 \\ 2 Dipartimento di Fisica and INFM, Universita' di Messina, I-98166, Messina, Italy. \\ ${ }^{3}$ Dipartimento di Scienze Fisiche, Università di Napoli "Federico II", \\ Complesso Universitario di Monte Sant'Angelo, via Cintia 80126 Napoli, Italy \\ ${ }^{4}$ Dipartimento di Ingegneria dell'Informazione, Seconda Università di Napoli, via Roma 29, 81031 Aversa (Caserta), Italy \\ ${ }^{5}$ Laboratoire des Verres, Université Montpellier II, 34095 Montpellier, France and \\ ${ }^{6}$ INFM Coherentia and Udr di Napoli, Napoli, Italy
}

(Dated: July 1, 2021)

\begin{abstract}
The increase of the viscosity, which is observed in attractive colloidal systems by varying the temperature or the volume fraction, can be related to the formation of structures due to particle aggregation. In particular we have studied the non trivial dependence of the viscosity from the temperature and the volume fraction in the copolymer-micellar system L64. The comparison of the experimental data with the results of numerical simulations in a simple model for gelation phenomena suggests that this intriguing behavior can be explained in terms of cluster formation and that this picture can be quite generally extended to other attractive colloidal systems.
\end{abstract}

PACS numbers: 83.80.Uv, 82.70.Dd, 83.60.Hc

Soft materials (polymers, proteins, colloids, etc.) represent a research field of large interest in science and technology and are a common domain for physics, chemistry and biology. The effective interaction amongst particles in these systems is in many cases the result of the competition between repulsive and attractive contributions and may give rise to aggregation processes with the formation of a variety of meta-stable or stable mesoscopic structures 1]-[4]. This is the case of gelation phenomena in attractive colloidal systems at low volume fractions $[\underline{6}-[\underline{9}]$. Here slow dynamics and eventually structural arrest are associated to the formation of long-living non-compact structures, with a change in the viscoelastic properties as the one typically observed in gelation phenomena. At present, this is a point of central interest in the study of attractive colloidal systems as the effects of aggregation processes at low volume fractions on the dynamics are still poorly understood [10-12.

A first interesting clue comes from the better known gel formation in polymer systems: here the phenomenology of gelation is typically interpreted in terms of a percolation transition [13]-15], that is the formation of a permanent spanning structure, accompanied by a structural arrest. This suggests that gelation phenomena could be understood in terms of formation of clusters, and eventually of a spanning structure, also in attractive colloidal systems. Here, on the other hand, at high volume fractions and low temperatures, structural arrest phenomena are observed with typical glassy features, that have been interpreted within the framework of the mode coupling theory [16, 17, 18]. In this case the glassy behavior can be strongly influenced by the short range attraction, but it cannot be simply described in terms of formation of clusters or of a percolating structure. Therefore the role of structure formation in the slow dynamics and the structural arrest of attractive colloidal systems is not com- pletely clear.

In this work we discuss the viscosity behavior in the copolymer micellar system $L 64$, where the short range attraction is due to an effective intermicellar interaction. We observe a transient gelation phenomenon followed by a structural arrest. For a fixed value of the temperature, as function of the volume fraction the viscosity increases, reaches a plateau and then increases again at higher volume fraction. In this process the rheological properties exhibit a crossover from gelation to a structural arrest typical of the glass transition. The comparison of the experimental findings with the numerical study of a simple model suggests that these two phenomena, which appear to be quite distinct, stem from two different mechanism: One related to the presence of long living clusters, the other due to crowding of particles. The model also suggests that the interrupted gel phenomena can be more generally related to colloidal gelation [ 6$]$ - [8] . In other colloidal systems, due to different features of the effective attractive interaction, the gelation phenomenon occurs at much lower temperature and volume fraction and it is more pronounced. Consequently, structural arrest and gelation almost coincide and occur close to the percolation transition. At higher temperatures clusters can be less persistent and structural arrest, occurring at higher volume fractions, exhibits features of the so called attractive glasses. Increasing further the temperature the effect of the bonding interaction vanishes and one finds features of the hard sphere glass transition [16, 17, 18.

We have studied the viscosity behavior, as a function of the temperature $T$ and of the micellar volume fraction $\Phi$, in the copolymer-micellar system L64 [19]-[23]. This is an aqueous solution of a non-ionic three-block copolymer made of polyethylene and polypropylene oxides, $\left[(P E O)_{13}-(P P O)_{30}-(P E O)_{13}\right]$ (L64 for short). These polymer molecules in water are $T$ dependent sur- 
factants that spontaneously form hydrated monodisperse spherical micelles in a wide $T-\Phi$ range. At low temperatures, both $P E O$ and $P P O$ are hydrophilic, so that the $L 64$ chains readily dissolve in water, and the polymer molecules exist as unimers. However, increasing $T, P P O$ tends to become less hydrophilic than $P E O$ creating an unbalance in the hydrophilicity between the central block and the end block of the polymer molecule that consequently acquires surfactant properties and self-assemble to form micelles. The inter-particle attraction is mainly due to the fact that, on further increasing $T$, water becomes progressively a poor solvent to both $P E O$ and $P P O$ chains. The phase diagram of the $L 64 / D_{2} O$ system is shown in Fig. 10 Due to the intermicellar attraction, the system has an inverted binodal curve with a lower consolute critical point, a $T-\Phi$ dependent percolation line where the viscosity exhibits a steep increase, and a glassy line as predicted by the mode coupling theory [19, 20]. In particular, by increasing the volume fraction the percolation line precedes the glassy line.

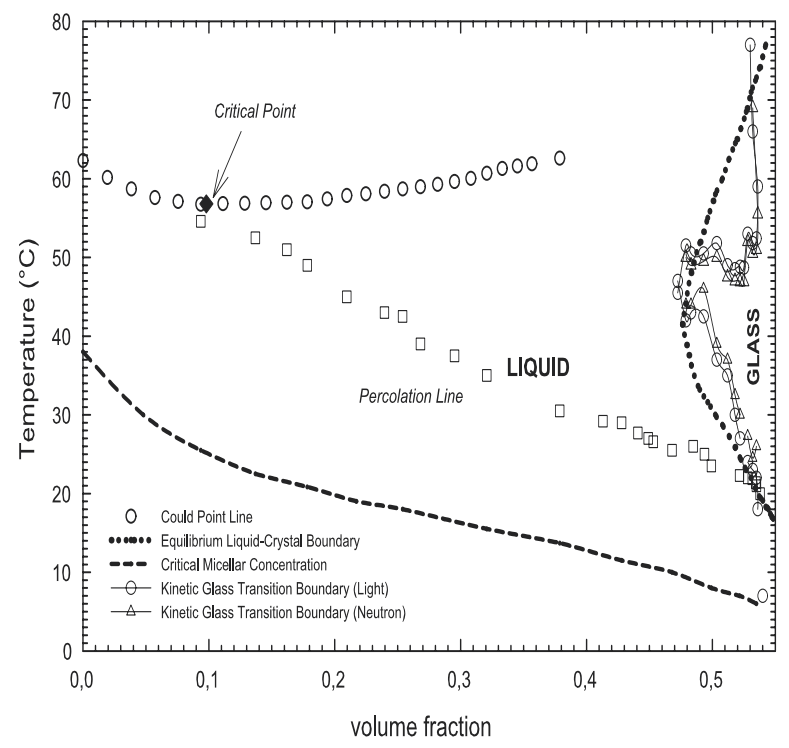

FIG. 1: The phase diagram of the $L 64 / D_{2} O$ system (adapted from Ref. [20]): the inverted binodal line (circles), the percolation line (empty squares) and the glassy line (circles and triangles on the right of the figure). The percolation and the glassy lines were measured by both quasi elastic light scattering and small angle neutron scattering.

Viscosity measurements, as a function of $T$ at different $\Phi$, were performed with a strain-controlled rheometer using a double wall Couette geometry, at the fixed frequency $\omega=1 \mathrm{sec}^{-1}$. To ensure a linear response, for different $T$ and $\Phi$, we have tested our system as a function of low applied strains, $\gamma$. After this check a low strain deformation, $\gamma=0.05$, was maintained for all the experiments. A crucial point is that the Peclet number
$P e=\dot{\gamma} \xi^{2} / 2 D_{0}$, which characterizes the amount of distortion of structures with linear dimension $\xi\left(D_{0}\right.$ is the particle short-time diffusion coefficient at low concentration where hydrodynamic effects dominate) must be small. In Ref. 21] it was found that $\xi \sim 5000 \stackrel{\circ}{A}$ and $D_{0}$ of the order of $2 \cdot 10 e^{-11} \mathrm{~m}^{2} / \mathrm{s}$ close to the percolation threshold, ensuring $P e<1$. These distortions are responsible for shear thinning in the system structures, which is expected to set on at $P e \approx 1$. In other words, the condition $P e<1$ constitutes an important experimental constraint in the study of the elasticity of systems characterized by clustering processes like polymer solutions and attractive colloids. In particular, this is true for viscosity measurements in phenomena dominated by long-range "critical" correlations, like those which originate the diverging incipient cluster typical of the percolation threshold in the sol-gel transition. Just in these processes, shear thinning can originate a "levelling off" of the measured viscosities.

In Fig. 2 the measured viscosity, $\eta$, of the system is plotted as a function of the temperature, $T$, at different volume fractions, $\Phi$. At each $\Phi, \eta$ is characterized by a growth from the values typical of the solvent $\left(\eta \sim 10^{-2} \mathrm{~Pa}\right)$ followed by a bending at high temperatures. At high $\Phi$ a second growth is observed in $\eta$ by further increasing the temperature: The first growth of the viscosity corresponds to the percolation transition (PT) 19], and the second growth to the structural arrest (SA) of the attractive glass transition 22].

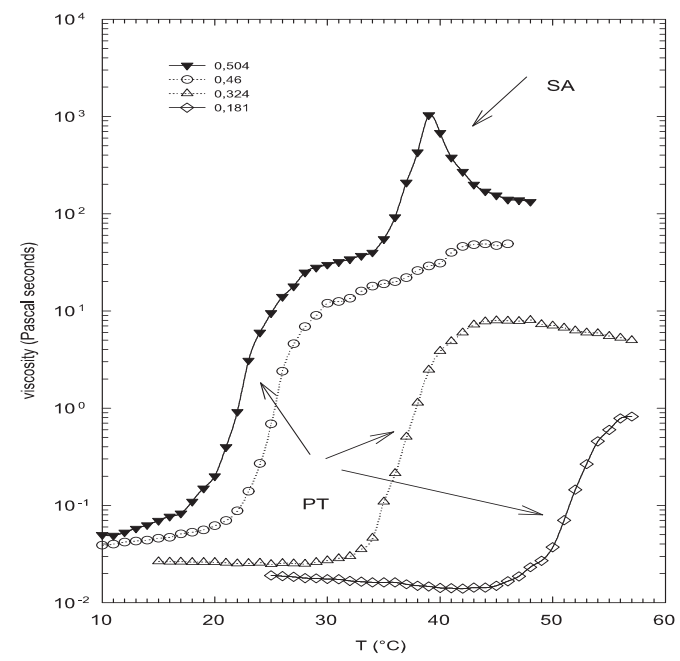

FIG. 2: The $L 64 / D_{2} O$ viscosity, $\eta$, as a function of the temperature, $T$, for different values of the volume fraction $\Phi$. In figure the percolation transition (PT) and the structural arrest $(\mathrm{SA})$ are shown.

In Fig. $3 \eta$ is plotted as a function of the volume fraction, $\Phi$, for different values of the temperature, $T$. At low 


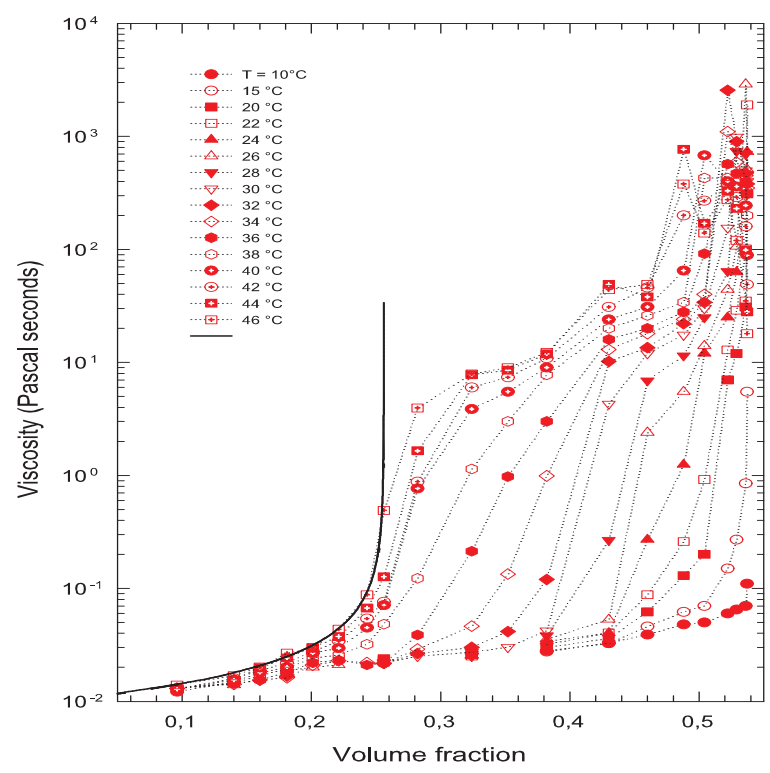

FIG. 3: The $L 64 / D_{2} O$ viscosity, $\eta$, as a function of the volume fraction, $\Phi$, for different values of the temperatures, $T$, in the range $10-46^{\circ} \mathrm{C}$. The continuous line is a power law, $\left(\Phi_{c}-\Phi\right)^{-k}$, with $k=1.35$.

volume fraction a first strong growth of the viscosity is found close to the percolation line. However, a bending in the viscosity is found, as the volume fraction is increased above the percolation threshold, and finally, by further increasing the volume fraction, a second growth corresponding to the glass transition [22] is observed. A similar effect in the viscosity behavior has also been observed in another micellar system in Ref. 24].

The strong growth of the viscosity close to the percolation line is reminiscent of the viscosity divergence observed in polymer gelation, and might be due to the presence of long living clusters in the system. The intermicellar interaction has an entropic origin. In fact, the micelle can be schematized as a hard sphere with polymers attached on the surface. When two micelles come close, the polymers get entangled (long-living bonds). Due to the entanglement, the two micelles have to overcome an entropic barrier to get separated. Therefore the bond lifetime $\tau_{b}$ will be related to the height of such entropic barrier $S_{b}$, i.e. $\tau_{b} \sim e^{S_{b}}$ (the entropy is in units of Boltzmann's constant). The other quantity of interest is the characteristic time $\tau_{u}$ for two close micelles to get entangled (bonded): $\tau_{u} \sim e^{S_{u}}$ where $S_{u}$ is the entropic barrier needed to be overcome to go from the un-bonded state to a bonded state. Therefore the probability $p_{b}$ for two close micelles to be bonded is given by

$$
p_{b}=\frac{e^{S_{b}}}{e^{S_{b}}+e^{S_{u}}} .
$$

Note that such bond probability can be quite smaller than 1, depending on the value of $S_{u}$. This could explain why in micellar systems percolation can occur at quite high volume fraction, as compared to other colloidal systems $[\underline{6}]-[\underline{9}]$.

To study the effect of aggregation and structural arrest in such micellar system, we propose a model which contains these two characteristic times for forming and breaking bonds. Tuning the parameters $\tau_{b}$ and $\tau_{u}$ we can study the gelation process with infinite bond lifetime (polymer gelation) and finite bond lifetime (micelles). A detailed discussion on the model and on the numerical simulations can be found in Ref. 25.

We consider a solution of tetrafunctional monomers with excluded volume interactions and performed Monte Carlo simulations on the cubic lattice. At $t=0$ the volume fraction $\Phi$ is fixed, and bonds between monomers are randomly quenched. The four possible bonds per monomers, randomly selected, are formed along lattice directions between monomers that are nearest neighbors and next nearest neighbors. In the case of polymer gelation, once formed the bonds are permanent. The monomer diffuse according to the bond-fluctuation dynamics (BFD) 26]. We let the monomer diffuse to reach the stationary state and then study the system for different values of the concentration. The system has a percolation transition at $\Phi_{c}=0.376 \pm 0.003$. In the case of colloidal gelation, we introduce a finite bond lifetime $\tau_{b}$. We start with the same configurations of the previous case, with a fixed $\Phi$ where the bonds have been randomly quenched. During the monomer diffusion with $B F D$ at each time step we attempt to break each bond with a frequency $1 / \tau_{b}$. Between monomers separated by a distance less than $l_{0}$ bonds are then formed with a frequency $1 / \tau_{u}$.

From the time autocorrelation function, $f_{\vec{q}}(t)$, of equilibrium density fluctuations with $\vec{q} \equiv\left(\frac{\pi}{4}, \frac{\pi}{4}, \frac{\pi}{4}\right)$, we calculate the relaxation time $\tau$ as the time such that $f_{\vec{q}}(\tau) \sim$ 0.1. In Fig. 4 the relaxation time, $\tau$, is plotted as function of the volume fraction, $\Phi$, for the permanent bonds and for the finite lifetime bonds case at different values of $\tau_{b}$. In the figure one finds the data for the permanent bond case on the left, and from left to right the data for finite bond lifetime, for decreasing values of $\tau_{b}$.

In the case of permanent bonds, $\tau(\Phi)$ displays a power law divergence, $\left(\Phi_{c}-\Phi\right)^{-k}$, at the percolation threshold, with $k=1.35$. For finite bond lifetime $\tau_{b}$ the relaxation time instead increases following the permanent bond case, up to some value $\Phi^{*}$ and then deviates from it. The longer the bond lifetime the higher $\Phi^{*}$ is. For higher $\Phi$ the increase of the relaxation time corresponds to the onset of the glassy regime in the relaxation behavior discussed in Ref. [25]. This qualitative behavior has also been confirmed via molecular dynamics simulations in Ref. 28]. This truncated critical behavior of the relaxation time qualitatively reproduces the experimental data shown in Fig. 3 In order to stress the analogy, we have plotted a power law behavior for the viscosity with 


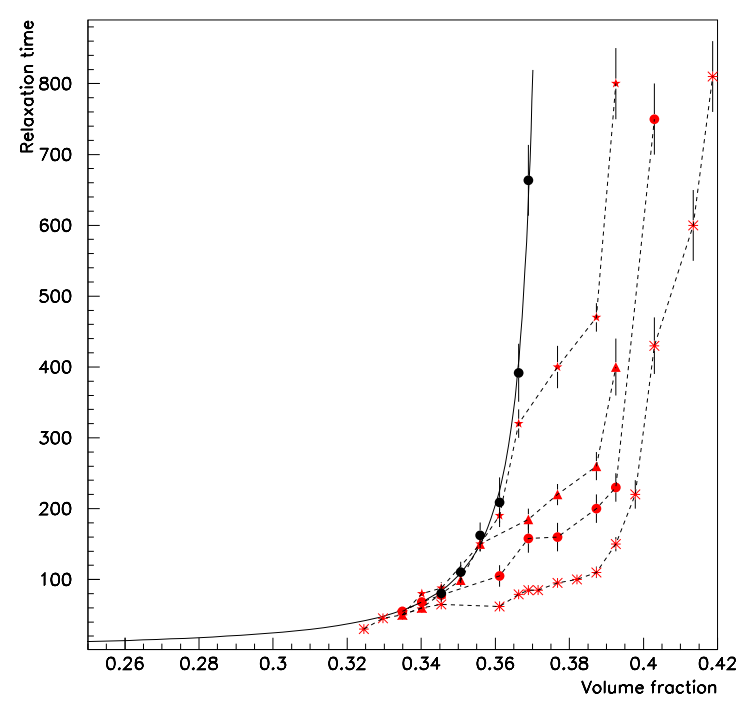

FIG. 4: The average relaxation time, $\tau$, as a function of the volume fraction, $\Phi$. From left to right: the data for the permanent bond case diverge at the percolation threshold with a power law (continuous line), see text. The other data refer to finite $\tau_{b}=3000,1000,400,100$ MCstep/particle decreasing from left to right (the dashed lines are guides to the eye). (Adapted from Ref. [25]).

the same value of $k=1.35$ (continuous curve in Fig. 31).

This comparison suggests the following interpretation for the experimental data. We consider that clusters of different sizes will be present in the system at any time $t$. In the permanent bond case, a cluster of radius $R$ diffuses amongst the others with a characteristic relaxation time, $\tau(R)$ : At the percolation threshold the connectedness length critically grows in the system and so does the overall relaxation time. In the case of a finite bond lifetime, $\tau_{b}$, there will be a cluster size $R^{*}$ so that $\tau_{b} \sim \tau\left(R^{*}\right)$. That is, clusters of size $R \leq R^{*}$ can be considered as permanent, whereas clusters of size $R>R^{*}$ will not contribute with their full size to the enhancement of the relaxation time in the system. Therefore, a long but finite bond lifetime produces the enhancement of the viscosity due to the formation of persistent clusters. On the other hand, it introduces an effective cluster size distribution with a cut-off which keeps the macroscopic viscosity finite in the system [29].

In conclusions, based on a minimal model for colloidal system we have analyzed the experimental viscosity data in copolymer-micellar systems. For a fixed temperature the viscosity exhibits a first steep growth as the volume fraction increases. This effect is due to coalescing clusters of monomers linked by long living bonds. Thus the viscosity increases as in a typical gelation phenomenon, but due to the finite lifetime of the bonds this increase is interrupted and followed by a saturation. As the volume fraction increases further, the viscosity exhibits a second steep growth until structural arrest is reached. The resulting glass transition can be related to the formation of a spanning cluster made of localized particles connected by bonds. It is the presence of the bonds which makes the difference between the attractive glass and a repulsive glass.

The research in Italy is supported by EU Network Number MRTN-CT-2003-504712, MIUR-PRIN 2004, MIUR-FIRB 2001, CRdC-AMRA, INFM-PCI. The research at MIT is supported by grant from Material Science Division of USA-DOE. We also acknowledge the EUMarie Curie Individual Fellowship HPMF-CI2002-01945.

[1] A. Stradner et al. Nature 432, 492 (2004).

[2] The Physics of Complex Systems, edited by F. Mallamace and H.E. Stanley (IOS Press, Amsterdam, 1997).

[3] F. Sciortino, Nature Materials, News and Views 1, 145 (2002).

[4] W.R. Chen, S.H. Chen and F. Mallamace, Science 300, 619 (2003).

[5] J. Groenewold and W.K. Kegel, J. Phys. Chem. B 105, 11702 (2001).

[6] A.D. Dinsmore and D.A. Weitz, J. Phys. : Condens. Matter 14, 7581 (2002).

[7] P.N. Segré et al., Phys. Rev. Lett. 86, 6042 (2001).

[8] A.I. Campbell et al., cond-mat/0412108

[9] S. Manley et al., cond-mat/0412716

[10] K. Kroy, M.E. Cates, and W.C.K. Poon, Phys. Rev. Lett. 92, 148302 (2004); A.M. Puertas, M. Fuchs, M.E. Cates, J. Chem. Phys. 1212813 (2004).

[11] F. Sciortino et al., Phys. Rev. Lett. 93, 055701 (2004).

[12] A. Coniglio et al., J. Phys. C: Condens. Matter 16, S4831 (2004).

[13] P.J. Flory, Principles of Polymer Chemistry, (Cornell University Press, 1953).

[14] P.G. de Gennes Scaling concepts in polymer physics, (Cornell University Press, 1980).

[15] D. Stauffer, A. Coniglio and M. Adam, Adv.in Polymer Sci. 44, 103 (1982).

[16] L. Fabbian et al., Phys. Rev. E 59, R1347 (1999); 60, 2430 (1999).

[17] J. Bergenholtz, M. Fuchs, and Th. Voigtmann, J. Phys. C: Condens. Matter 12, 6575 (2000).

[18] K. A. Dawson et al., Phys. Rev. E 63, 11401 (2000).

[19] L. Lobry et al., Phys. Rev E 60, 7076 (1999).

[20] W.R. Chen, S.H. Chen and F. Mallamace, Phys. Rev E 66, 021403 (2002); W.R. Chen et al., Phys. Rev E 68, 041402 (2003).

[21] C. Liao et al., J. Appl. Cryst. 33, 677 (2000); S.-H. Chen et al., Coll. and Surfaces A 95, 183-185 (2001).

[22] F. Mallamace et al., J. Phys. C: Condens. Matter, in press.

[23] F. Mallamace et al., Phys. Rev. Lett. 84, 5431 (2000).

[24] F. Laflèche, D. Durand and T. Nicolai, Macromolecules 36, 1331 (2003); F. Laflèche, D. Durand and T. Nicolai, Macromolecules 36, 1341 (2003). 
[25] E. Del Gado et al., Europhys. Lett. 63, 1 (2003); Phys. Rev. E 69, 051103 (2004).

[26] I. Carmesin and K. Kremer, Macromolecules 21, 2819 (1988).

[27] E. Del Gado, L. de Arcangelis and A. Coniglio, Europ.
Phys. J. E 2, 359 (2000); Phys. Rev E 65, 041803 (2002).

[28] I. Saika-Voivod et al., Phys. Rev. E 70, 041401 (2004).

[29] A. Coniglio, J. Phys. C: Condens. Matter 13, 9039 (2001). 